\title{
Scalable Photonic Quantum Computation through Cavity-Assisted Interactions
}

\author{
L.-M. Duan ${ }^{1,2}$ and H. J. Kimble ${ }^{3}$ \\ ${ }^{1}$ FOCUS Center and MCTP, Department of Physics, University of Michigan, Ann Arbor, Michigan 48109-1120, USA \\ ${ }^{2}$ Laboratory of Quantum Information, USTC, Hefei, Anhui 230026, China \\ ${ }^{3}$ Norman Bridge Laboratory of Physics 12-33, California Institute of Technology, Pasadena, California 91125, USA
}

(Received 25 September 2003; published 25 March 2004)

\begin{abstract}
We propose a scheme for scalable photonic quantum computation based on cavity-assisted interaction between single-photon pulses. The prototypical quantum controlled phase-flip gate between the singlephoton pulses is achieved by successively reflecting them from an optical cavity with a single-trapped atom. Our proposed protocol is shown to be robust to practical noise and experimental imperfections in current cavity-QED setups.
\end{abstract}

PACS numbers: 03.67.Pp, 03.67.Lx, 42.50.Pq

Realization of quantum computation requires accurate coherent control of a set of qubits. A small volume optical cavity provides a platform to achieve strong coherent interactions between atoms and photons, and has been exploited as the critical component in several schemes for implementation of quantum computation and communication [1-4]. In a prototypical cavity-based quantum computation scheme of Ref. [1], the atoms are adopted as qubits while photons mediate the interaction between them. Scaling to large-scale quantum computation via this paradigm then requires that many atoms be localized and separately addressed within a tiny optical cavity [1], or alternatively be coherently transported into and out of the cavity mode [5]. However, in spite of recent significant laboratory advances [5-9], these tasks remain daunting experimental challenges.

Here, we propose a scalable quantum computation scheme where qubits are encoded as polarizations of single-photon pulses. An optical cavity with a singletrapped atom is employed as the critical resource to achieve controlled gate operations between photonic qubits and to act as a high efficiency single-photon detector. The proposed computation architecture is based on the state-of-the-art in cavity quantum electrodynamics [6], can be readily scaled up to many qubits, and could be integrated with protocols for the realization of quantum networks [2].

Quantum computation with single-photon polarizations as qubits $[10,11]$ has the obvious advantage that the number of qubits can readily be scaled up by generating many single-photon pulses. The main obstacle to this approach is that it is exceedingly difficult to achieve quantum gate operations between single-photon pulses. The typical photon-photon coupling rate in available materials is orders of magnitude too small to allow for any meaningful gate operation at the single-quantum level. An interesting idea, as has been put forward recently in the so-called linear optics quantum computation scheme [12], is to achieve effective nonlinear interaction between photons through feed forward from high efficiency single-photon detectors. Though this approach is a very important advance, a significant obstacle is that the required efficiency $\alpha$ of the single-photon detectors for scalable quantum computing is extremely high (e.g., for gate success with probability $p \simeq 0.99, \alpha \gtrsim$ 0.999987 [13]).

In our proposed scheme, we combine the advantage of scalability from the photonic qubits and the power of strong atom-photon coupling in a high-finesse optical resonator. Such a cavity with one or few atoms in a configuration of far-off-resonant interactions provides an effective Kerr nonlinearity for the input light $[11,14,15]$, as was first observed in Ref. [11]. However, this nonlinear phase shift is typically too small for realization of the operation of the prototypical quantum controlled-NOT gate (C-NOT). Compared with the approach of Ref. [11], our new protocol has the following significant advances: (i) A different interaction mechanism between photon pulses leads to a much larger effective interaction rate sufficient for the realization of a quantum C-NOT gate with current experimental capabilities. (ii) The conditional phase flip in our scheme is very insensitive to variation of the atom-photon coupling rate, so that high-fidelity gate operations can be realized even if the atom is not localized in the Lamb-Dicke regime. (iii) The pulse shapes for pairs of interacting single photons suffer very small changes due to interactions with the atom-cavity system, which is otherwise quite difficult to achieve [15]. (iv) Finally, the noise properties of our scheme are quite favorable, and should allow significant improvement in the error threshold for large-scale, faulttolerant quantum computation.

The basis states for our qubit consist of two orthogonal polarization states of a single-photon pulse, denoted by $|h\rangle$ and $|v\rangle$. A series of single-photon pulses is generated by emission from a single atom in a cavity $[2,16]$; singlequbit operations on these photonic qubits are accurately performed through polarization rotations. The critical problem for quantum computation with these qubits is to achieve a nontrivial two-qubit interaction. Here, we 
choose the quantum controlled phase-flip (CPF), where the CPF gate for qubits $j$ and $k$ flips the phase of the input state if both qubits are in $|h\rangle$ polarizations, and has no effect otherwise. The CPF gates, together with simple single-qubit operations, realize universal quantum computation [17].

As illustrated in Fig. 1(a), the CPF gate for two arbitrary pulses $j$ and $k$ is implemented by simply reflecting them successively from a high- $Q$ cavity which contains a single-trapped atom. The atom has three relevant levels as shown in Fig. 1(b), and is initially prepared in an equal superposition of the two ground states, i.e., $\left|\Phi_{a i}\right\rangle=$ $(|0\rangle+|1\rangle) / \sqrt{2}$. The atomic transition $|1\rangle \longrightarrow|e\rangle$ is resonantly coupled to a cavity mode $a_{h}$, which has $h$ polarization and is resonantly driven by the $h$ polarization component of the input single-photon pulse. The $v$ polarization component of the input pulse is reflected by the mirror $\mathrm{M}$.

Before describing the detailed model and supporting calculations, first we summarize the basic ideas of our scheme, which consists of two critical steps. (A) By reflecting one single-photon pulse, say $j$, from the cavity and the mirror, a CPF gate between the atom and the pulse $j$ is achieved as described by the unitary operator $U_{a j}^{C P F}=$ $e^{i \pi|0\rangle_{a}\langle 0|\otimes| h\rangle_{j}\langle h|}$. (B) A composition of the CPF gates between the atom and the pulses $j, k$ generates a CPF gate between the pulses $j$ and $k$ described by the unitary operator $U_{j k}^{C P F}=e^{i \pi|h\rangle_{j}\langle h|\otimes| h\rangle_{k}\langle h|}$, while restoring the atom into its initial state $\left|\Phi_{a i}\right\rangle$. Experimentally the composition is performed by successively "bouncing" the pulses from the cavity [see Fig. 1(a)].

Step $(A)$ - When the incoming photon is $v$ polarized, it will be reflected by the mirror $M$ without any phase and shape change. When the incoming photon is in $h$ polarization, it is resonant with the bare cavity mode if the atom is in the $|0\rangle$ state and thus acquires a phase of $e^{i \pi}$ after its reflection; however, if the atom is in the $|1\rangle$ state, the frequency of the dressed cavity mode from the resonant atom-cavity coupling is significantly detuned from

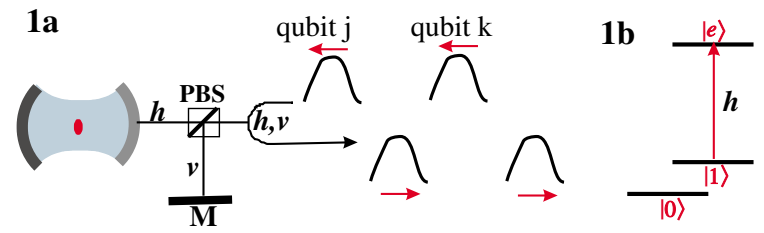

FIG. 1 (color online). (a) Schematic setup to implement the controlled phase flip (CPF) gate between two single-photon pulses $j$ and $k$. With a polarization beam splitter (PBS), the $h$-polarized component of the single-photon pulse is reflected by the cavity, while the $v$-polarized component is reflected via the mirror M. The optical paths from the polarization beam splitter (PBS) to the cavity and to the mirror $\mathrm{M}$ are assumed to be equal. (b) The relevant level structure of the atom trapped in the cavity (e.g., the states $|0\rangle$ and $|1\rangle$ could denote hyperfine states of an alkali atom in the ground-state manifold while $|e\rangle$ is an excited state). the frequency of the incoming pulse. In this case, the cavity functions in the same fashion as the mirror $\mathrm{M}$ and the photon pulse is reflected without a phase change. A composition of the above subprocesses realizes the desired CPF gate $U_{a j}^{C P F}$ between the atom and the photon.

Step $(B)$ - Critical to the second step of our protocol is the following operator identity:

$$
\begin{aligned}
U_{j k}^{C P F}\left|\Psi_{j k}\right\rangle \otimes\left|\Phi_{a i}\right\rangle= & U_{a j}^{C P F} R_{a}(-\pi / 2) U_{a k}^{C P F} R_{a}(\pi / 2) \\
& \times U_{a j}^{C P F}\left|\Psi_{j k}\right\rangle \otimes\left|\Phi_{a i}\right\rangle,
\end{aligned}
$$

where $\left|\Psi_{j k}\right\rangle$ denotes an arbitrary state of the photonic qubits $j$ and $k$, and $R_{a}(\theta)$ is a single-bit rotation on the atom which transforms according to $R_{a}(\theta)|0\rangle=$ $\cos \theta / 2|0\rangle+\sin \theta / 2|1\rangle \quad$ and $\quad R_{a}(\theta)|1\rangle=-\sin \theta / 2|0\rangle+$ $\cos \theta / 2|1\rangle$. The identity (1) demonstrates that the CPF gate between two arbitrary single-photon pulses $j$ and $k$ can be implemented by first reflecting the pulse $j$ from the cavity as shown in Fig. 1(a), then applying a ( $\pi / 2)$-pulse laser on the atom, then reflecting the pulse $k$ from the cavity, then applying a $(-\pi / 2)$-pulse laser on the atom, and finally reflecting the pulse $j$ again from the cavity.

The CPF gate $U_{a j}^{C P F}$ between the atom and the photon pulse can also be used to achieve quantum nondemolition (QND) measurement of the photon number in the pulse. For this purpose, we simply prepare the atom in the state $\left|\Phi_{a i}\right\rangle$, reflect the to-be-measured photon pulse from the cavity, apply a $R_{a}(\pi / 2)$ rotation on the atom, and finally perform a measurement of the atomic state in the basis $\{|0\rangle,|1\rangle\}$. The measurement outcome is " 0 " if and only if the $h$ component of the pulse has a photon. By the same avenue, we can also measure the parity of several photonic qubits ("parity" concerns whether a series of pulses has a total even or odd photon number in their $h$ components) by successively reflecting them from the cavity, and can as well measure the total photon number of both $h$ and $v$ components of a single pulse by reflecting it twice from the cavity with a polarization flip between the two reflections. Such QND measurements have wide applications for quantum information processing $[18,19]$. Note that the measurement of atomic internal states can be done with near $100 \%$ efficiency through the quantum jump technique [4]. So, the efficiency of our QND measurement is principally only limited by the inefficiency of the CPF gate between the atom and the photon pulse caused by atomic spontaneous emission loss, which as we will see later, is significantly less than the inefficiency of conventional destructive single-photon detectors.

Now we present a detailed theoretical model to demonstrate that the CPF gate $U_{a j}^{C P F}$ between the atom and the single-photon pulse $j$ can be obtained simply by reflecting the latter from the cavity. The initial state of the pulse $j$ can be expressed as $\left|\Psi_{p}\right\rangle_{j}=c_{h j}|h\rangle_{j}+c_{v j}|v\rangle_{j}$, where $c_{h j}$ and $c_{v j}$ are arbitrary superposition coefficients. The polarization component states $|\mu\rangle_{j}(\mu=h, v)$ have the form $|\mu\rangle_{j}=\int_{0}^{T} f_{j}(t) a_{\mu}^{\text {in } \dagger}(t) d t|\mathrm{vac}\rangle$, where $f_{j}(t)$ is the normalized pulse shape as a function of time $t, T$ is the pulse 
duration, $a_{\mu}^{\text {in }}(t)$ are one-dimensional field operators (cavity input operators) with the standard commutation relations $\left[a_{\mu}^{\mathrm{in}}(t), a_{\mu^{\prime}}^{\mathrm{in \dagger}}\left(t^{\prime}\right)\right]=\delta_{\mu \mu^{\prime}} \delta\left(t-t^{\prime}\right)$ [20], and $|\mathrm{vac}\rangle$ denotes the vacuum of all the optical modes. The cavity mode $a_{h}$ is driven by the corresponding cavity input operator $a_{h}^{\text {in }}(t)$ through [20]

$$
\dot{a}_{h}=-i\left[a_{h}, H\right]-(i \Delta+\kappa / 2) a_{h}-\sqrt{\kappa} a_{h}^{\text {in }}(t),
$$

where $\kappa$ is the cavity (energy) decay rate and the Hamiltonian

$$
H=\hbar g\left(|e\rangle\left\langle 1\left|a_{h}+\right| 1\right\rangle\langle e| a_{h}^{\dagger}\right)
$$

describes the coherent interaction between the atom and the cavity mode $a_{h}$. The detuning $\Delta$ in Eq. (2) is meant to be 0 for our scheme, but we retain it here for subsequent pedagogical purposes. The cavity output $a_{h}^{\text {out }}(t)$ is connected with the input by the standard input-output relation

$$
a_{h}^{\text {out }}(t)=a_{h}^{\text {in }}(t)+\sqrt{\kappa} a_{h} .
$$

As the $v$ component of the pulse is reflected by the mirror $\mathrm{M}$, we simply have $a_{v}^{\text {out }}(t)=a_{v}^{\text {in }}(t)$.

Equations (2)-(4) determine the evolution of the joint state of atom and photon pulse, and can be solved without further approximation through numerical simulation. However, before presenting the simulation results, first we attack this problem analytically with some rough approximations to reveal the underlying physics. If the atom is in the state $|0\rangle$, the Hamiltonian $H$ does not play a role in Eq. (2). In this case, from Eqs. (2) and (4) we find

$$
a_{h}^{\text {out }}(t) \approx \frac{i \Delta-\kappa / 2}{i \Delta+\kappa / 2} a_{h}^{\text {in }}(t),
$$

where the high-frequency components of the field operators $a_{\mu}^{\text {in }}(t)$ and $a_{\mu}^{\text {out }}(t)$ have been discarded, which is a valid approximation if the input pulse shape $f_{j}(t)$ changes slowly with time $t$ compared with the cavity decay rate, i.e., $\left|\partial_{t} f_{j}(t) / f_{j}(t)\right| \ll \kappa$. Under this approximation, we have $a_{h}^{\text {out }}(t) \approx-a_{h}^{\text {in }}(t)$ for resonant interaction $\Delta=0$, so the $h$ component acquires the phase $\pi$ after reflection from the cavity. However, if the atom is in the state $|1\rangle$, the response function of the cavity is modified by the coupling (3), where for the case of strong coupling [21], the two dressed cavity modes have frequencies that are effectively detuned from that of the input pulse by $\Delta=$ $\pm g$, respectively. In the case that $g \gg \kappa$, we have $a_{h}^{\text {out }}(t) \approx a_{h}^{\text {in }}(t)$ from Eq. (5), thereby confirming the preceding analysis to give the desired CPF gate $U_{a j}^{C P F}$.

Armed with this understanding, we finally present exact numerical simulations for the theoretical model described by Eqs. (2)-(4). In the simulation, we discretize the continuum field operators $a_{h}^{\text {in }}(t)$ and $a_{h}^{\text {out }}(t)$, and change the dynamics into the Schrödinger picture to avoid operator ordering. The details of the simulation method can be found in Ref. [22]. Atomic spontaneous emission noise is effectively described by an imaginary part $\left(-i \gamma_{s} / 2\right)(|e\rangle\langle e|-| 1\rangle\langle 1|)$ in the Hamiltonian $H$ [22], where $\gamma_{s}$ is the spontaneous emission rate from the state $|e\rangle$. For convenience, the input pulse is taken to be Gaussian with $f_{j}(t) \propto \exp \left[-(t-T / 2)^{2} /(T / 5)^{2}\right]$, where $t$ ranges from 0 to $T$ [23].

The numerical simulations show that the CPF gate $U_{a j}^{C P F}$ works remarkably well. First of all, the conditional phase factor is either $e^{i \pi}$ or $e^{i 0}$ depending on the atomic state $|0\rangle$ or $|1\rangle$, and this phase factor is very insensitive to the variation of the coupling rate $g$ in the typical parameter region. For instance, its variation is smaller than $10^{-6}$ for $g$ varying from $6 \kappa$ to $\kappa$. This result cannot be understood naively from Eq. (5), from which one gets a phase of $e^{i 0}$ only when $g \gg \kappa$. The reason for this discrepancy is that we have two addressed cavity modes with symmetric effective detunings $\Delta= \pm g$, and their joint effect makes the phase factor $e^{i 0}$ very stable even if $g$ is reduced to a value comparable with $\kappa$. The stability of the conditional phase against variations of $g$ in the typical parameter region is an important advantage of our scheme, as $g$ in current experiments suffers significant random variation (roughly by a factor of 2 ) due to residual atomic motion [6].

The simulation also shows that the output pulse basically has the same shape as the input pulse if the pulse duration $T \gg 1 / \kappa$. Figure 2(a) shows the output pulse shapes $\left|f_{i}(t)\right|$ for the cases of the atomic states $|1\rangle$ and $|0\rangle$, respectively, and demonstrates very good overlap with the input pulse shape shown in the same figure. In more quantitative terms, we consider the fidelity $F$ of the CPF gate $U_{a j}^{C P F}$ for the input atom-photon state $\left|\Phi_{a i}\right\rangle \otimes$ $\left[\left|\Psi_{p i}\right\rangle=(|h\rangle+|v\rangle) / \sqrt{2}\right]$. Reductions in $F$ below unity are caused by shape mismatching between the input and the output pulses and can be numerically calculated. Figure 2(b) shows the gate fidelity $F$ calculated in this
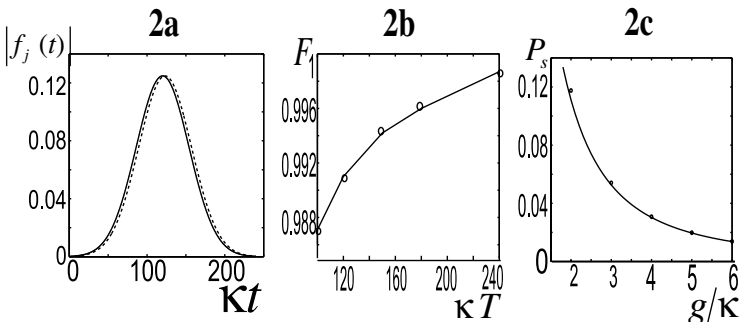

FIG. 2 (color online). (a) The shape functions $\left|f_{j}(t)\right|$ for the input pulse (solid curve) and the reflected pulse with the atom in the state $|0\rangle$ (dashed curve) and $|1\rangle$ (dotted curve), respectively. The dotted and solid curves closely match and are hardly distinguishable in the figure. (b) Fidelity $F$ due to shape mismatch for the quantum CPF gate as a function of the input pulse duration $T$ in units of $\kappa^{-1}$. The gate fidelity quickly approaches 1 for $\kappa T \gg 1$. (c) The probability $P_{s}$ of spontaneous emission loss versus the normalized cavity coupling rate $g / \kappa$, assuming $\gamma_{s}=\kappa$ (circles). The solid curve shows the fit by the empirical formula $P_{s} \approx 1 /\left(1+2 g^{2} / \kappa \gamma_{s}\right)$. Other parameters for (a), (b), $g=3 \kappa, \gamma_{s}=\kappa, \Delta=0$, and, for (c), $T / 5=$ $24 / \kappa, \Delta=0$. 
way for different pulse durations $T$. For $T=240 / \kappa$ (corresponding to a pulse width $T / 5 \sim 1 \mu \mathrm{s}$ for the parameters of Ref. [6]), the gate fidelity is about $99.9 \%$. The shape of the output pulse is also very insensitive to variation of the coupling rate $g$ in the typical parameter region. For instance, the relative shape change is smaller than $10^{-4}$ for $g$ varying from $6 \kappa$ to $\kappa$.

The dominant noise in our CPF gate arises from photon loss due to atomic spontaneous emission [24], leading to a vacuum-state output when the input is a single-photon pulse. This noise yields a leakage error (also called an erasure error) which means that the final state is outside of the qubit Hilbert space $\{|h\rangle,|v\rangle\}$ [17]. Figure 2(c) shows the probability $P_{s}$ of spontaneous emission loss as a function of $g / \kappa$ for the input state $|1\rangle \otimes|h\rangle$, assuming $\gamma_{s}=\kappa$. The curve is well simulated by the empirical formula $P_{s} \approx 1 /\left(1+2 g^{2} / \kappa \gamma_{s}\right)$. If the initial state of the system is $\left|\Phi_{a i}\right\rangle \otimes\left|\Psi_{p i}\right\rangle$, the average probability of the leakage error per $U_{a j}^{C P F}$ gate is given by $P_{e}=P_{s} / 4$. In current experiments [6], typically $\left(\kappa, \gamma_{s}\right) / 2 \pi \approx$ $(8,5.2)$, and $g / 2 \pi \approx 25 \mathrm{MHz}$, which yields $P_{e} \approx 0.8 \%$. With these parameters, a typical pulse width $T / 5 \approx$ $24 / \kappa \approx 0.5 \mu \mathrm{s}$. As the pulses $j$ and $k$ are injected successively for the CPF gate $U_{j k}^{C P F}$, we need to introduce a time delay of few $\mu$ s between them. For demonstration-ofprinciple experiments, this time delay can be routinely achieved through simple fiber loops. To obtain longer time delay, atomic ensembles could be employed to store photon pulses for several seconds [25-27].

Because the principal noise in our scheme is photon loss during gate operations which is modeled as a leakage error, very efficient quantum error correcting codes can be incorporated into this computation scheme to achieve fault-tolerance [17]. For instance, a rough estimate in Ref. [28] shows that through concatenated coding, quantum computation can tolerate leakage error at a percent level per gate, as compared to the error threshold of about $10^{-5}$ for general quantum errors [17]. The leakage error only affects the probability to register a photon from each pulse and has no influence on the fidelity of its polarization state if a photon is registered for each qubit (e.g., through QND or destructive measurements). So, leakage error induces small inefficiency for each gate (at a level of a few percent), which is not debilitating for experimental quantum computing up to dozens of CPF gates even without quantum error correction.

In summary, we have shown that a cavity with a singletrapped atom, conventionally used as a single-photon source, can be exploited to realize scalable, fully functional quantum computation. The proposed scheme is well based on the state-of-the-art in cavity quantum electrodynamics, is robust to various experimental sources of noise, and offers a promising approach to the realization of large-scale fault-tolerant quantum computation.

This work was supported by the Michigan startup fund, the FOCUS seed funding, the CSF, the CAS, the
“97.3" project No. 2001CB309300, the MURI Center for Quantum Networks (No. DAAD19-00-1-0374), the NSF (No. EIA-0086038 and No. PHY-0140355), and the Office of Naval Research (No. N00014-02-1-0828).

[1] T. Pellizzari, S. A. Gardiner, J. I. Cirac, and P. Zoller, Phys. Rev. Lett. 75, 3788 (1995).

[2] J. I. Cirac, P. Zoller, H. J. Kimble, and H. Mabuchi, Phys. Rev. Lett. 78, 3221 (1997).

[3] M. S. Zubairy, M. Kim, and M. O. Scully, Phys. Rev. A 68, 033820 (2003).

[4] For a review, see C. Monroe, Nature (London) 416, 238 (2002).

[5] J. A. Sauer et al., quant-ph/0309052.

[6] J. McKeever et al., Phys. Rev. Lett. 90, 133602 (2003); J. McKeever et al., Nature (London) 425, 268 (2003).

[7] M. Keller et al., Appl. Phys. B 76, 125 (2003).

[8] M. A. Rowe et al., Quantum. Inf. Comp. 2, 257 (2002).

[9] A. Kuhn, M. Hennrich, and G. Rempe, Phys. Rev. Lett. 89, 067901 (2002).

[10] I. L. Chuang and Y. Yamamoto, Phys. Rev. A 52, 3489 (1995).

[11] Q. A. Turchette et al., Phys. Rev. Lett. 75, 4710 (1995).

[12] E. Knill, R. Laflamme, and G. Milburn, Nature (London) 409, 46 (2001).

[13] S. Glancy, J. M. LoSecco, H. M. Vasconcelos, and C. E. Tanner, Phys. Rev. A 65, 062317 (2002).

[14] A. Imamoglu, H. Schmidt, G. Woods, and M. Deutsch, Phys. Rev. Lett. 79, 1467 (1997).

[15] K. M. Gheri, K. Ellinger, T. Pellizzari, and P. Zoller, Fortschr. Phys. 46, 401 (1998).

[16] C. K. Law and H. J. Kimble, J. Mod. Opt. 44, 2067 (1997).

[17] J. Preskill, Lecture Notes on Quantum Computation, see http://www.theory.caltech.edu/people/preskill/ph229

[18] K. Banaszek and K. Wodkiewicz, Phys. Rev. Lett. 82, 2009 (1999).

[19] L.-M. Duan, G. Giedke, J. I. Cirac, and P. Zoller, Phys. Rev. Lett. 84, 4002 (2000).

[20] D. F. Walls, and G. J. Milburn, Quantum Optics (SpringerVerlag, Berlin, 1994).

[21] H. J. Kimble, Phys. Scr. T76, 127 (1998).

[22] L.-M. Duan, A. Kuzmich, H. J. Kimble, Phys. Rev. A 67, 032305 (2003).

[23] The exact shape of the input pulse is actually not important if the shape changing is sufficiently slow compared to the cavity decay rate $\kappa$.

[24] The photon loss could be also from absorption and scattering by the cavity mirrors, and their contributions (typically small) can be included through a modified atomic spontaneous emission rate.

[25] M. Fleischhauer and M. D. Lukin, Phys. Rev. Lett. 84, 5094 (2000).

[26] L. M. Duan, M. D. Lukin, J. I. Cirac, and P. Zoller, Nature (London) 414, 413 (2001).

[27] A. Kuzmich et al., Nature (London) 423, 731 (2003).

[28] E. Knill, R. Laflamme, and G. J. Milburn, quant-ph/ 0006120 . 